Questions vives

\section{Questions Vives}

Recherches en éducation

$\mathrm{N}^{\circ} 27 \mid 2017$

(Re) découvrir John Dewey : quelle actualité en formation des adultes?

\title{
À la recherche d'une expérience esthétique en musique : analyse d'une action didactique conjointe autour de la recherche d'un son "musical" pour une production collective au piano \\ Pascale Batézat-Batellier
}

\section{(2) OpenEdition \\ Journals}

\section{Édition électronique}

URL : https://journals.openedition.org/questionsvives/2137

DOI : 10.4000/questionsvives. 2137

ISSN : $1775-433 \mathrm{X}$

\section{Éditeur}

Université Aix-Marseille (AMU)

\section{Édition imprimée}

Date de publication : 31 juillet 2017

ISBN : 978-2-912643-51-3

ISSN : 1635-4079

\section{Référence électronique}

Pascale Batézat-Batellier, « À la recherche d'une expérience esthétique en musique : analyse d'une action didactique conjointe autour de la recherche d'un son "musical" pour une production collective au piano », Questions Vives [En ligne], № 27 | 2017, mis en ligne le 31 décembre 2017, consulté le 21 septembre 2021. URL : http://journals.openedition.org/questionsvives/2137 ; DOI : https://doi.org/ 10.4000/questionsvives.2137

Ce document a été généré automatiquement le 21 septembre 2021.

\section{cc) (†)}

Questions Vives est mis à disposition selon les termes de la licence Creative Commons Attribution Pas d'Utilisation Commerciale - Pas de Modification 4.0 International. 


\title{
À la recherche d'une expérience esthétique en musique : analyse d'une action didactique conjointe autour de la recherche d'un son "musical" pour une production collective au piano
}

\author{
Pascale Batézat-Batellier
}

\section{Introduction}

1 Cet article s'intéresse aux effets d'une première appréhension d'un langage musical, par des élèves de huit à dix ans. Les élèves abordent un répertoire qui dérange leurs habitudes de jeu et d'écoute musicale ${ }^{1}$. Les cours observés ont été filmés et accompagnés d'entretiens avec l'enseignant. Ils ont lieu dans un conservatoire de région, sont collectifs (trois à six élèves), durent chacun six semaines et s'ajoutent au cours individuel. Nos observations portent sur deux cours de six séances chacun, soit deux groupes d'élèves, un par cours. Le professeur prend comme référence des œuvres de Cowell (1897-1965), Kurtág (1926-) et Crumb (1929-) qui représentent tout un pan de la musique des XXe et $\mathrm{XXI}^{e}$ siècles communément appelé musique « contemporaine $»^{2}$. Son objectif premier est bien d'ouvrir "l'imaginaire sonore " des élèves et non de leur apprendre à jouer une œuvre de l'un de ces compositeurs.

On peut émettre l'hypothèse qu'il s'agit d'une source d'expérience et d'apprentissage (Dewey, 1938) inaugurale et originale dont on escompte une production musicale spécifique, avec des moyens corporels propres aux élèves et dans une esthétique qui ne leur est pas familière. 
3 Notre problématique consiste d'une part de donner à voir et à comprendre i) comment le professeur initie chez l'élève cette expérience esthétique de la recherche d'un son;ii) comment le jeune élève garde en mémoire les sensations corporelles (kinesthésiques et auditives) qu'il aura eues lors de ces expériences; iii) comment la mémoire corporelle engage un processus de dévolution chez l'élève dans l'action didactique conjointe.

Notre recherche s'appuie sur la Théorie de l'Action Conjointe en Didactique (Sensevy \& Mercier, 2007 ; Sensevy, 2011) dans une perspective anthropologique, en filiation avec la Théorie Anthropologique du Didactique (Chevallard, 1992 et sous presse) et avec la Théorie des Situations Didactique (Brousseau, 1998). Elle tente d'articuler ces concepts didactiques avec les paradigmes de l'esthétique du son associé à la dimension interprétative d'une œuvre musicale. Elle pose la question d'une forme de parenté entre le processus de dévolution (Brousseau, 1998; Sensevy, 2011) et celui de l'interprétation. Il s'agit donc de décrire l'action des professeurs et des élèves dans cette situation conjointe.

5 La démarche est ethnographique (Lévi-Strauss, 1949; Bazin, 2000) dans le sens où elle décrit l'action elle-même, in situ et s'inscrit dans une clinique du didactique (Leutenegger 2000), au sens où il s'agit d'une démarche à la fois ascendante et attentive aux détails, dans une acception proche de celle de Foucault (1963) concernant la clinique médicale, et de celle de Ginzburg (1989) proposant un paradigme indiciaire.

\section{Références culturelles et paradigmes en jeu}

6 Cette partie a pour objectif de décrire succinctement les notions et les modes de jeu que la professeure ${ }^{3}$ aborde avec les élèves.

7 Nous présenterons et situerons les compositeurs que la professeure donne à écouter aux élèves. « Le choix des œuvres et des compositeurs est justement lié aux sons que l'on va utiliser après » dit-elle ${ }^{4}$. Elle part de ses objectifs d'apprentissage qu'elle introduit en donnant des exemples musicaux, et non l'inverse. C'est-à-dire, que l'objectif n'est pas de connaitre tel ou tel compositeur, mais bien de savoir jouer à partir de certains éléments d'un tel style de musique. Dans notre analyse, la professeure s'attache à certains traits caractéristiques qui font appel à des techniques de jeu particulières que nous décrivons ci-après.

\subsection{Styles et techniques de jeu}

8 Un style de musique, dans le sens de Goodman (1984/1996) est constitué de traits caractéristiques d'un ensemble d'œuvres. Ici nous avons affaire à un pan de la musique de piano des $\mathrm{XX}^{\mathrm{e}}$ et $\mathrm{XXI}^{\mathrm{e}}$ siècles. Si la genèse de ces techniques se situe autour de 1912 avec The tides of Manaunaum de Cowell ${ }^{5}$ (1897-1965) où celui-ci invente le cluster, elles continuent à interroger les compositeurs que la professeure a fait écouter aux élèves : Kurtág (1926-), et Crumb (1929-) .

9 La professeure fait écouter à ses élèves The fairy answer (1929) de Cowell. Cette œuvre introduit un mode de jeu, le «string piano», jeu dans les cordes du piano. Inventé par Cowell, ce style de jeu en appelant d'autres évoluera vers le " piano préparé ». Ensuite la professeure propose les Játékok (composés entre 1973 et 2010) de Kurtág. Les Játékok ont d'abord été conçu comme un recueil pédagogique pour le piano. Ils sont devenus depuis « une encyclopédie de la pensée de Kurtág » (Wilheim, s.d.). Pour Crumb (1929-), qui est le 
troisième compositeur écouté, la démarche est différente. D’après Poirier (2015) : «plus que le langage, qui n'est pas la préoccupation première du compositeur, c'est le rapport à l'instrument ou à la voix et la réflexion sur le rôle de l'exécutant qui caractérise la place que Crumb a occupée à partir des années soixante ». Ce qui, comme nous le verrons plus tard, correspond pour une part à la démarche du professeur ${ }^{6}$. Certains traits caractéristiques des œuvres écoutées montrent que l'écriture de ces compositeurs s'oppose à l'écriture pianistique classique et romantique (contrapunctiques, mélodiques, harmoniques) ${ }^{7}$ et aux «manières » de produire des sons (utilisation du clavier et des pédales uniquement). Ils expérimentent une autre façon d'utiliser le piano (photo 1) : en jouant par exemple dans les cordes comme avec une harpe (b), en bloquant des cordes avec une gomme (a) et en jouant avec le clavier pour changer le son de la corde frappée, en frappant le bois du piano comme une percussion, en posant, sur les cordes préalablement frappées, des feuilles de papier pour les faire vibrer ${ }^{8}$.

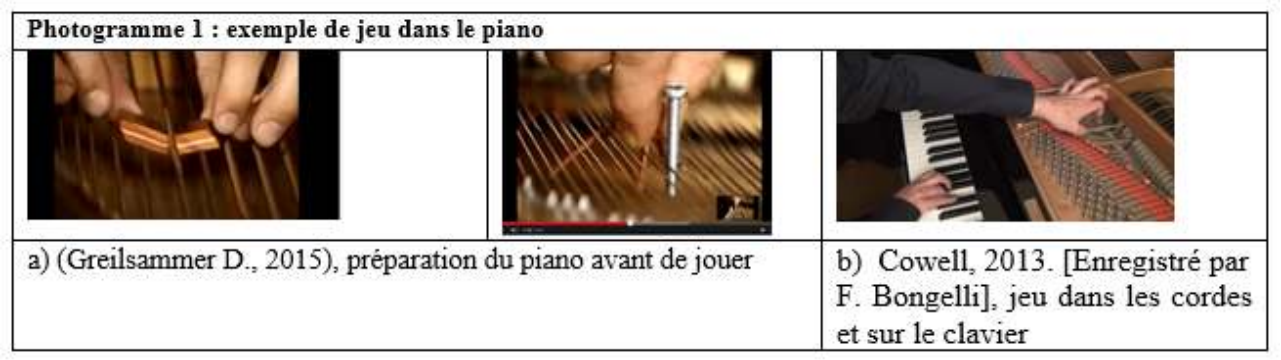

Les cours étudiés ici sont fondés sur une approche orale non codée, improvisée et expérimentée. L'écriture n'existe que par le fait que la professeure s'y réfère (cf. entretien avec la professeure ci-dessus), mais l'élève n'y a pas accès. L'objectif final, formulé ${ }^{9}$ par la professeure et qui s'observe pendant les séances, est la construction d'une production musicale dans le style de jeu cité. Cette construction suppose une transposition didactique des pratiques savantes évoquées plus haut (Verret, 1975 ; Chevallard, 1991). Les traits caractéristiques de ce style qui ont été retenus par la professeure et les élèves sont : sur le clavier, le cluster et le glissando ${ }^{10}$; dans les cordes, l'utilisant d'objets et le glissando ; l'utilisation de la pédale de résonance. C'est pourquoi elle prend comme une exemplification (Goodman, 1984) les auteurs de référence. Comme l'écrit P (e-mail du 16-01-2016) :

Le choix des œuvres et des compositeurs est justement lié aux sons que l'on va utiliser après et va du plus facile à ressentir pour eux jusqu'au plus éloigné de leur univers (je trouve)

- Objet trouvé et jeu de paume de Kurtag sont tirés des jatekok et parlent facilement à leur imaginaire: cela utilise comme matériau (vocabulaire) les glissandos sur clavier, les clusters avec différents touchers et nuances ainsi que la résonnance de la pédale. Le fait que des clusters peuvent être joués de différentes manières montrent que cela dépend de l'histoire que l'on veut raconter.

- the fairy answer de Cowell utilise une phrase jouée d'une part sur le clavier et d'autre part en réponse avec les glissandos sur cordes et pizz de cordes dans le piano : Cowell est le 1er compositeur à être allé dans le piano (je crois). La forme question-réponse permet aux enfants d'identifier les différents matériaux.

- Crumb dans la continuité de Cowell est celui qui a utilisé le piano dans toutes ses possibilités : la pièce tirée des Macrocosmos a un côté un peu mystique mais parle à l'imaginaire, dans le fantastique et les couleurs sonores. Les matériaux utilisés sont très diversifiés : jeu au clavier avec cordes bloquées, objet dans le piano (chaîne), glissandos sur cordes dans différentes tessitures, clusters, utilisation de toutes les tessitures notamment extrêmes (grand espace sonore). 
11 Dans ces exemples, nous pouvons également considérer qu'elle propose un style de pensée (Fleck, 2005) qui devra être partagé par le collectif lors des transactions didactiques et de leur évolution dans la mésogenèse ${ }^{11}$. «Pour "agir en première personne", l'élève devra assumer, à un certain moment, une forme de solitude dans l'apprentissage : l'enfant qui apprend à marcher devra accepter de lâcher la main qu'on lui tend. C'est cette notion que Brousseau (1998) a très tôt définie comme dévolution» (Sensevy, 2011, p. 74). Le processus de dévolution suppose un milieu adéquat qui permette à l'élève de construire son expérience à des fins d'apprentissage comme le signifie Dewey (1938/1993) :

Pour nous, puisque toute connaissance particulière se présente comme le résultat d'une enquête particulière, la conception de la connaissance en soi ne peut qu'être une généralisation des propriétés dont on a découvert qu'elles appartiennent aux conclusions qui sont les résultats de l'enquête. La connaissance, en tant que terme abstrait, est le nom du produit des enquêtes bien menées. ${ }^{12}$ (Dewey, 1938/1993, p. 65)

Dans le sens où, nous le préciserons plus loin, l'expérience est propre à l'individu, elle existe quand celui-ci est confronté à une résistance (milieu qui fait problème). Cette résistance peut être ici identifiée en rapport avec le jeu collectif et les interactions entre élèves et professeur et entre les élèves eux-mêmes, mais aussi en rapport avec les parties du corps de l'élève qui sont mobilisées pour jouer et l'instrument de musique. Ce que Dewey (1938/1993) caractérisait comme "déséquilibre » dans la situation existe bien lorsque la professeure dévolue à l'élève la responsabilité de son apprentissage. Ce processus de dévolution est en rapport avec le jeu collectif et les interactions entre élèves et professeur et entre les élèves eux-mêmes. La place du professeur et de l'élève ( topogénèse) dans ce processus évoluera au fur et à mesure de l'avancée des savoirs.

\subsection{De l'esthétique}

13 Il s'agit aussi de construire une perception de ce qui fait référence dans cette musique, c'est-à-dire, en reprenant dans une acception très large l'étymologie du mot

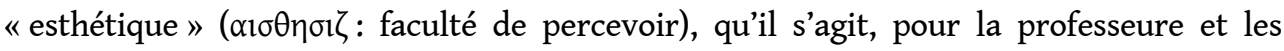
élèves, de travailler sur la perception de ce qui caractérise ce style de jeu pianistique (Goodman, 1984) et les sons qui sont utilisés.

Il s'agit de travailler sur l'esthétique des sons qui sont utilisés dans ce courant musical en faisant écho à Nattiez (1987, p. 34), pour qui « la jouissance, la contemplation ou la lecture de l'œuvre, l'interprétation musicale ainsi que les approches scientifiques et analytiques de la musique sont situées de facto du côté de l'esthétique. » Pour Dewey (1938/1993), le mot «artistique » fait référence à l'acte de production, le mot " esthétique » à l'acte de perception et de plaisir. La phase esthétique est donc celle où l'on éprouve, elle est réceptive, et évaluative, elle va de l'extérieur de l'être vers l'intérieur de l'être. Dewey précise qu'il n'y a cependant pas de terme (mot) pour signifier le plaisir dans l'acte de production : le processus de l'art qui se situe sur le plan de la production (œuvre d'art), a un lien organique avec l'esthétique qui se situe elle-même sur le plan de la perception. La perception, c'est l'étude de l'objet. Elle est constamment présente - de façon continue ou ponctuelle en fonction des moments - dans l'acte de création. Pour Dewey elle en permet la reconstruction comme une "conscience animée "; c'est une boucle qui se construit pendant tout le processus de création, jusqu'à l'obtention d'une satisfaction de la part de 
l'artiste ; notamment parce qu'elle permet à l'artiste d'évaluer l'avancée de sa création, d'en éprouver du plaisir. Elle permet aussi aux auditeurs ou spectateurs d'apprécier (en dehors d'un jugement de valeur) l'œuvre avec laquelle ils sont en contact et d'en éprouver du plaisir ou non. La démarche que la professeure souhaite faire adopter aux élèves nous semble assez proche de celle décrite ci-dessus.

Les paradigmes de l'esthétique des sons dans les cours observés concernent les attaques, grain, souffle, sons déterminés et indéterminés comme les compositeurs le demandent dans une légende souvent jointe à la partition (fig. 1 on frappe avec le poing sur le clavier). Dans les séances observées, le rapport à la partition n'existe pas pour les élèves. La figure 1, ci-dessous, illustre le «comment il faut faire " connue par P. Nous avons remarqué qu'elle le transmettra aux élèves oralement.

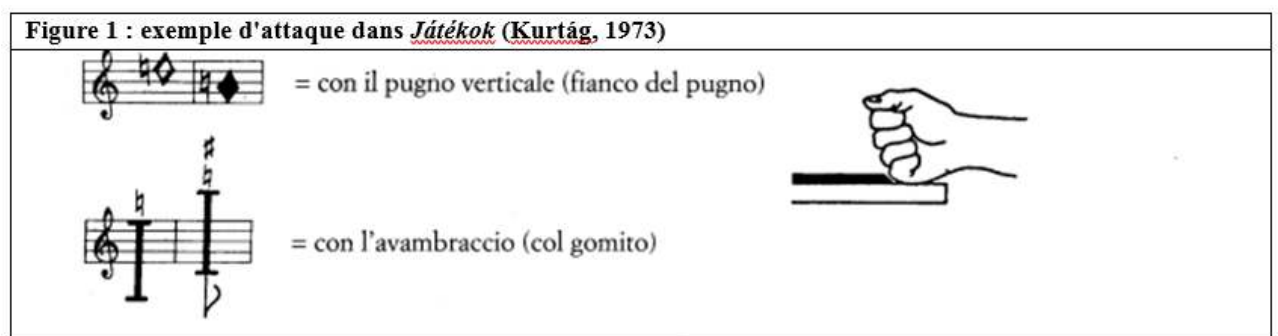

\subsection{De l'interprétation}

La notion d'interprétation n'apparaît stricto sensu qu'au XIX siècle (Caullier, 2012). Elle fait l'objet de plusieurs définitions. Certains instrumentistes pensent qu'il faut être fidèle au compositeur, mais insistent sur le fait de créer chez le public des émotions et donc d'avoir une «intervention personnelle » dans la façon de jouer. Pour Menuhin (1976, p. 329), l'interprète se situe «entre le compositeur et le public, il apporte l'élément vivant, il donne le battement de la vie à la sécheresse des notes alignées sur la portée " grâce à une maitrise technique et une lecture sensible de l'œuvre. Badura-Skoda présente ainsi le travail de l'interprète: "il commente, il communique, il traduit, qu'il en ait conscience ou non » (Badura-Skoda \& Badura-Skoda, 1974, p. 13).

Les musicologues insistent sur le fait qu'interpréter demande une connaissance de l'époque de l'œuvre jouée, du compositeur et du style de jeu qui convient (Arnold, 1983/1993 ; Donington, 1998). Cependant, une conception nouvelle de l'interprète voit le jour dans le monde contemporain (Caullier, 2012) qui inclut le recours à l'improvisation libre ${ }^{13}$. Celle-ci « déplace la focalisation musicologique traditionnelle, de l'objet-partition (et de la figure du compositeur) vers l'objet-phonogramme (et la figure de l'interprète) [... ] ». Alors, comme pour les élèves de cette étude, « bien des repères familiers vacillent ou disparaissent lorsqu'on quitte le monde du texte pour celui du son » (IRCAM, 2010).

On peut déjà dire que le "référent » (la connaissance de l'époque de l'œuvre, la référence musicale, les modes de jeux) produit le "déjà-là » du contrat didactique, et que le milieuproblème est celui de l'œuvre à interpréter (qu'on soit, interprète ou compositeurinterprète, ou improvisateur etc.).

Si dans l'interprétation d'une œuvre, il y a des connaissances acquises (style, esthétique, époque, forme), une manière de comprendre l'œuvre (jusque dans les moments d'improvisation demandés par certains compositeurs contemporains) et de la rendre intelligible, une manière de faire (technique) ${ }^{14}$, alors il y a une prise de responsabilité 
dans l'exécution d'une œuvre. Dans tous les cas, l'interprète assume une part essentielle de l'accès à l'œuvre musicale. Notre hypothèse de travail est qu'un processus de dévolution , voulu par le compositeur, s'instaure. C'est une partie de ce que vivent les élèvesmusiciens lorsque, après les avoir orientés vers une situation précise (mésogenèse), la professeure se retire progressivement en leur laissant une forme de liberté dans le jeu pianistique. Dans ce processus de dévolution :

L'Élève doit aussi accepter, avec la clause proprio motu, cette forme de désengagement du Professeur : il doit accepter d'être à certains moments laissé à lui-même, savoir que l'appropriation du savoir passe par une forme d'indépendance et de solitude, de détachement nécessaire des paroles et des actes du Professeur. » (Sensevy, 2011a, p. 75).

Dans l'interprétation, les choix des professeurs et des élèves prennent en compte les références culturelles acquises. Ces choix sont induits par les phénomènes techniques de facture instrumentale ${ }^{15}$, et les limites instrumentales qui diffèrent d'un instrument à un autre.

Ici, la professeure (P) prendra aussi appui sur les capacités de techniques instrumentales acquises antérieurement par les élèves. C'est le «déjà-là » du contrat didactique (Brousseau, 1998; Sensevy, 2011a). Les élèves ont déjà effectué un an de piano (ils savent jouer des mélodies très simples avec leurs doigts sur un clavier, ils savent lire une partition simple avec des noires des blanches et des croches). P leur demande d'aller, d'une certaine manière, à l'encontre de ces capacités pour tenter une démarche d'exploration sonore proche de celle des compositeurs cités plus haut. Ces capacités sont celles-là mêmes du contrat didactique de la première année de piano de ces élèves. Elles permettent de se reposer sur un système d'habitudes institutionnelles qui, paradoxalement, peut freiner de nouvelles expériences. C'est aussi la rupture de ce contrat implicite qui le rend visible (Brousseau, 1998) au chercheur. Cette rupture permet d'amener les élèves à concevoir une approche de la musique autre que celle à laquelle ils sont habitués (musiques dites "classique » ou «de variété » dont le point commun est qu'elles sont, pour la plupart, tonales et mesurées) et de leur permettre de nouvelles expériences sonores.

\section{Problématique, particularité du cadre théorique et de la méthodologie}

\subsection{Problématique}

Les cours collectifs observés s'ajoutent au cours individuel plutôt tourné vers des œuvres allant du XVII ${ }^{e}$ au début du XXe siècle, avec une technique pianistique de clavier. Dans ce contexte, quand les enseignants proposent aux élèves de jouer dans le piano avec des ustensiles, ils proposent un milieu (Brousseau, 2003) qui, par les problèmes qu'il pose, crée un environnement favorable à l'expérimentation d'un nouveau répertoire. Les enseignants sont amenés à faire des choix, c'est pourquoi ce qu'ils proposent aux élèves n'est pas toujours complètement exploité. Cependant, par les possibilités que ce milieu offre, certains savoirs non prévus par l'enseignant sont abordés. C'est le cœur de notre problématique : 
professeur puisse, dans un processus de dévolution, le laisser responsable de son propre apprentissage en prenant comme "prétexte" celui d'avoir à interpréter la musique qui aura été inventée collectivement avec ses pairs? Il s'agit alors de savoir s'il existe une parenté entre le processus de dévolution et celui d'interprétation.

Cette problématique est posée par tout un processus que nous souhaitons rendre visible en gardant en mémoire les questions suivantes :

- si pour travailler ce style de musique dite "contemporaine", il faut aborder une esthétique du son qui lui corresponde ; si la finalité de l'exploration sonore ${ }^{16}$ est que, ce qui a l'air mécanique dans le piano soit « dé-mécanisé »; si cela donne à l'élève l'occasion de chercher des timbres jusqu'alors inconnus de lui ; alors, comment l'élève aborde-t-il l'apprentissage du jeu pianistique avec son corps dans cette expérience gestuelle et sensitive?

26 - Comment formuler «le problème musical» posé pas la professeure dans son aménagement du milieu? Et, dans ce contexte, lorsque $\mathrm{P}$ dévolue à un élève la responsabilité de trouver une solution à ce "problème musical ", est-ce que ce dernier, s'il l'accepte et l'assume, entre dans un processus d'interprétation?

27 Nous pouvons maintenant, en utilisant les termes didactiques précédemment définis, poser l'hypothèse suivante: la dévolution, par la prise de responsabilité qu'elle induit, participe à la construction de la fonction d'interprète chez l'élève.

\subsection{Cadre théorique, méthode et représentation de l'action}

Comme présenté dans l'introduction, nous nous plaçons dans la lignée de Brousseau (1998), Chevallard, (1992 et sous presse) et plus particulièrement dans celle de la TACD avec Sensevy \& Mercier (2007), Sensevy (2011). Cette dernière théorie est peu exploitée en musique. Certains chercheurs ont articulé les concepts de transposition didactique (Beaugé, 2002 ; Bourg, 2008), ceux de jeux, d'équilibration didactique contrat-milieu par l'action conjointe professeur-élève, de double sémiose (Batézat-Batellier, 2012 ; Forest \& Batézat-Batellier, 2013) et de dévolution (Mili, 2012). Bourg et Guillot (2015) dans l'article où ils présentent plusieurs théories convoquées pour l'analyse de l'enseignement musical, étudient de façon succincte la pertinence de la TACD dans certaines situations. Ici, nous souhaitons continuer à la mettre à l'épreuve.

29 La démarche est de type ethnographique (Lévi-Strauss, 1949 ; Bazin, 2000) ${ }^{17}$ dans le sens où elle décrit l'action elle-même, in situ, dans l'observation participante de cette activité. Toutefois, la posture du chercheur est « dedans-dehors », car s'il est présent derrière la caméra et s'il peut orienter les entretiens qui ont lieu avant et après les séances, il n'intervient pas pendant les séances. Notre démarche s'inscrit également dans une clinique du didactique (Leutenegger, 2000). Dans cette étude, nous complétons notre démarche par l'idée que pour décrire une approche esthétique «il s'agit d'indiquer certains symptômes de la façon dont une œuvre d'art fonctionne comme symbole " (Goodman, 1984/1996, p. 152). Nous proposons d'étendre ce raisonnement à une méthodologie en didactique de la musique: "nous devons partir de la pratique ordinaire puis nous essayons d'ajuster une formule à la pratique. Nous pouvons alors réviser la formule, réviser la pratique ou les deux » (Goodman, 1984/1996, p. 151-152).

C'est donc en partant d'une description contextualisée de la pratique de musiciens professionnels, de l'observation de celle des élèves, en faisant des allers et retours entre 
leur propre expérience (Dewey, 1938/1993) et cette pratique professionnelle, que nous pourrons analyser les processus d'appropriation d'une esthétique pianistique chez des élèves débutants.

31 Filmer les cours permet en outre de capturer dans leur nature même les dimensions analogiques de l'action qui sont analysées ici, notamment celle du corps. Pour rester au plus près des intentions du professeur, des entretiens ont lieu avant et après les séances si nécessaire. Cependant certains entretiens avec l'enseignante ont été très courts, ils ont été complétés par des courriels. Sur les six séances de cours de chacun des groupes observés, les séances 1,2 , et 6 ont été filmées (sachant que le concert d'élève a lieu pendant la sixième séance). Les élèves ont signé deux autorisations de diffusion des images (celle du chercheur prévu pour diffusion dans les instances de la recherche, et celle demandée par le conservatoire, prévue pour diffusion dans la presse). Les autres séances n'ont été filmées pour laisser les élèves libres pendant leur invention. Nous avons cependant été présent et avons pris des notes. Pour l'analyse des données nous procédons à des analyses épistémiques, analyses a priori, transcription des séances et réduction sous forme de synopsis. Enfin, des photogrammes au sein de représentation hybrides, texteimage (Forest, 2009 ; Sensevy, 2011b) participent à la description.

\section{Description et Analyse}

\subsection{Analyse a priori}

Une analyse a priori est une description structurée des phénomènes didactiques qui peuvent advenir dans une situation donnée (Assude \& Mercier 2007). Le tableau 1, cidessous, a pour objectif de rendre visible un certain nombre de phénomènes didactiques à partir des catégories théoriques que nous utilisons en fonction d'une situation donnée et des enjeux de savoirs. Dans la colonne de gauche nous avons indiqué les catégories théoriques d'actions qui nous semblent pertinentes pour la suite de l'étude et dans celle de droite les actions des élèves et/ou du professeur et/ou ce sur quoi ils agissent. 


\begin{tabular}{|c|c|c|}
\hline \multicolumn{3}{|c|}{$\begin{array}{l}\text { Tableau } 1 \text { : analyse a priori des cours de piano en groupe restreint } \\
\text { Caractéristique de la Situation d'apprentissage : Faire improviser et inventer une musique à } \\
\text { partir d'un jeu pianistique du type «piano préparé ». }\end{array}$} \\
\hline \multicolumn{2}{|c|}{ Catégories } & $\begin{array}{l}\text { Ce que font les élèves et/ou la professeure } \\
\text { Ce sur quoi ils agissent }\end{array}$ \\
\hline \multirow{2}{*}{ 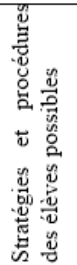 } & $\begin{array}{l}\text { Le déjà-là du } \\
\text { contrat didac- } \\
\text { tique }\end{array}$ & $\begin{array}{l}\text { - Savoir écouter } \\
\text { - Connaitre le clavier du piano }\end{array}$ \\
\hline & En construction & $\begin{array}{l}\text { - Écouter des professionnels de référence ("savoir savant") } \\
\text { - Faire décrire verbalement par les élèves ce qu'ils entendent } \\
\text { - Faire des essais de jeu sur le piano } \\
\text { - Inventer une histoire servant de support à l'invention musicale }\end{array}$ \\
\hline \multicolumn{2}{|c|}{$\begin{array}{l}\text { Possibles sources de ré- } \\
\text { troactions du milieu }\end{array}$} & $\begin{array}{l}\text { - Le son } \\
\text { - Le corps de l'élève } \\
\text { - Les réactions des autres élèves } \\
\text { - le « piano préparé » }\end{array}$ \\
\hline \multicolumn{2}{|c|}{$\begin{array}{l}\text { Action du professeur pour } \\
\text { définir, ou réguler le jeu } \\
\text { d'apprentissage }\end{array}$} & $\begin{array}{l}\text { - Fait écouter des enregistrements audio } \\
\text { - Propose des objets } \\
\text { - Oriente les élèves par des questions } \\
\text { - Montre des exemples sonores, en jouant. }\end{array}$ \\
\hline \multicolumn{2}{|c|}{$\begin{array}{l}\text { Modes de validation pos- } \\
\text { sibles }\end{array}$} & $\begin{array}{l}\text { Par l'élève : } \\
\text { - son, écoute et sensation qu'il perçoit ; } \\
\text { - verbalisation de ce qui est entendu ou fait ; } \\
\text { Par la professeure : } \\
\text { - confirmation de ce que font ou disent les élèves. }\end{array}$ \\
\hline \multicolumn{2}{|c|}{$\begin{array}{l}\text { Savoir et connaissances } \\
\text { en jeu : capacités épisté- } \\
\text { miques qui émergent de la } \\
\text { pratique conjointe. }\end{array}$} & $\begin{array}{l}\text { - Identifier un style de musique } \\
\text { - Découvrir le fonctionnement intérieur du piano } \\
\text { - Faire le rapport entre : le geste et le son et/ou un matériau } \\
\text { choisi et le son } \\
\text { - Être responsable de ses choix et de la production sonore qui en } \\
\text { résulte. }\end{array}$ \\
\hline
\end{tabular}

Ce tableau nous donnera des points de repères pour appréhender l'analyse de cas qui suit.

\section{2. Écoute-découverte et exploration sonore sur le piano}

Nous avons choisi deux groupes, des cinq groupes de six élèves étudiés, qui nous paraissaient susceptibles de mieux donner à voir les phénomènes que nous voulons analyser. Les élèves du premier groupe sont nommés par des lettres (EA) et les élèves du deuxième groupe par des chiffres (E3). Nous nommons les groupes en fonction de l'ordre chronologique de l'année scolaire et non en fonction de leur apparition dans notre analyse. La professeure est nommée P. Son objectif d'écoute est : « Le choix des œuvres et des compositeurs est justement lié aux sons que l'on va utiliser après et va du plus facile à ressentir pour eux jusqu'au plus éloigné de leur univers » (e-mail du 16-01-2016). Voici ce que nous avons observé de sa démarche car elle n'a pas été décrite par la professeure ellemême. Les cours sont construits plus ou moins de la même façon :

\section{Séance 1}

- écoute silencieuse de 3 extraits d'œuvres enregistrées: Játékok de Kurtág, 2006; Makrokosmos de Crumb, 2004 ; The fairy answer de Cowell, 2013.

- Exploration sur le piano des différents sons entendus, puis de différents sons possibles à l'initiative de l'enseignante, invention musicale.

Séances 2, 3, 4, 5

- rappel oral de ce qui a été fait la semaine précédente ;

42 - répétition de la création des élèves ;

43 - audition publique. 


\section{2.a Constitution de références et expérience kinesthésique} avec des ustensiles).

\section{Séance 1, découverte \\ Phase 1 découverte des différents sons possibles}

Après avoir situé sur une frise chronologique la période étudiée, la professeure (appelée « $\mathrm{P} »)$ fait écouter les œuvres citées plus haut. $\mathrm{P}$ demande aux élèves de deviner ce qu'ils entendent, et comment c'est joué.

Lorsque le professeur de Formation Musical (FM) fait écouter l'enregistrement d'une œuvre, dans le conservatoire dans lequel les élèves étudient, il leur demande, entr'autre, de reconnaitre les instruments entendus ${ }^{18}$. Aussi le contrat didactique antérieur " contrat d'écoute » peut-il être spécifié de la façon suivante : « écouter en silence, et deviner quels sont les instruments qui jouent ». Il y a là une forme habituelle de transaction avec $\mathrm{P}$ à propos d'un contenu musical.

Or dans cette phase les élèves sont déroutés, car il y a rupture d'une partie du contrat. La forme d'interaction de la transaction reste, mais le contenu change. Ce qu'ils écoutent ne correspond pas à ce qu'ils ont l'habitude d'entendre ou de travailler. Le nouveau contrat n'est pas explicité, l'implicite reste : «écoutez en silence " mais il ne s'agit pas là de deviner quels sont les instruments joués (contrat d'écoute en F.M.) puisqu'il n'y a qu'un seul instrument et que les élèves ne le savent pas encore. La nouveauté de ce contrat didactique se révèle au fur et à mesure des questions que $\mathrm{P}$ posera. Elle essaie de construire une nouvelle référence culturelle pour les enfants, en utilisant la stratégie suivante: elle leur indique un chemin qui fait appel à différents sens connus d'eux : visuel, kinésique, auditif puis à un univers plutôt discursif ne leur disant : «je vais vous faire écouter celui-là et, en plus vous allez imaginer un titre ou une histoire ou je ne sais quoi là-dessus » ( $1^{\text {ère }}$ séance deuxième groupe). « Rassurés » les élèves peuvent répondre. Mais ils restent gênés quand il faut reconnaître les sons. La rupture de contrat est plus frappante : ils cherchent dans ce qu'ils savent déjà et qui est très ancré, c'est-à-dire «à quel instrument cela correspond » mais ne trouvent pas.

En instaurant un élément nouveau (l'écoute des enregistrements de musique « contemporaine »), $\mathrm{P}$ introduit un nouveau type de problème au sein du contrat d'écoute. $\mathrm{P}$ procède ainsi : partant d'un contrat connu (reconnaître des instruments à l'écoute) elle pose un problème en proposant un milieu inconnu (le jeu pianistique dans les cordes et

Quand ils sont à court d'idées, elle leur donne des pistes et leur fait réécouter les enregistrements. Puis en confirmant leurs dires elle confirme le nouveau savoir (institutionnalisation des savoirs, transcript 1, 18'50 ci-dessous). 


\begin{tabular}{|c|c|c|}
\hline \multicolumn{3}{|c|}{ Transcript 1 : institutionnalisation des savoirs, $1^{\text {re }}$ séance $2^{\mathrm{e}}$ groupe } \\
\hline Time code & & discours \\
\hline & E & du piano, la pédale et la voix \\
\hline \multirow[t]{3}{*}{18700} & $\mathrm{P}$ & $\begin{array}{l}\text { la voix bravo, alors vous avez trouvé beaucoup de choses. Donc on va récapituler. Quels sont les } \\
\text { instruments ou l'instrument que vous avez entendu? }\end{array}$ \\
\hline & E & du piano? \\
\hline & $\mathrm{P}$ & du piano (elle confirme) \\
\hline 18,13 & $\mathrm{E}$ & des cordes? \\
\hline $18^{\prime} 14$ & $\mathrm{P}$ & des cordes du piano \\
\hline \multirow[t]{2}{*}{$18^{\prime} 16$} & $\mathrm{E}$ & une voix \\
\hline & $\mathrm{P}$ & une voix, la pédale du piano \\
\hline \multirow[t]{2}{*}{$18 \cdot 19$} & & $\mathrm{Ha}$ ! la pédale, c'est tout \\
\hline & $\mathrm{P}$ & il y avait un bruit bizarre à un moment donné tu m'as dit (...) \\
\hline 18,30 & $\mathrm{P}$ & $\begin{array}{l}\text { elle refait écouter l'extrait où on entend les bruits de papier d'aluminium qui vibre sur les cordes. } \\
\text { Mais ils ne trouvent pas. Elle ne donne pas la solution }\end{array}$ \\
\hline
\end{tabular}

Dans le transcript 2 ci-dessous, la professeure montre quelques façons de jouer, en rapport avec les sonorités entendues dans les enregistrements, puis propose aux élèves d'essayer.

\begin{tabular}{|c|c|c|}
\hline \multicolumn{3}{|c|}{ Transcript 2 : introduction de la phase recherche sonore, $1^{\text {re }}$ séance du $2^{e}$ groupe } \\
\hline $18 \div 50$ & $\mathrm{P}$ & $\begin{array}{l}\text { Dans tout ce qu'on a entendu, même quand il y a des sons de harpes ou des sons bizarres c'est } \\
\text { toujours du piano. On va s'amuser à trouver des bruits bizarres... } \\
\text { Vous allez voir comment [...] }\end{array}$ \\
\hline $19 \cdot 30$ & $\mathrm{P}$ & $\begin{array}{l}\text { Alors lả tout de suite je vais vous montrer comment on fait les sons bizarres qu'on a entendus. Allez, } \\
\text { on y va! }\end{array}$ \\
\hline
\end{tabular}

\section{Expérience kinesthésique}

\section{Phase 2 : exploration sonore sur le piano.}

$P$ introduit l'idée que les élèves vont travailler sur des sons qui sont nouveaux pour eux. En se référant aux enregistrements, elle ouvre les possibles et leur propose d'autres façons de faire, avec ou sans ustensiles, dans les cordes ou sur le clavier. Elle fait essayer les pédales aux élèves, montre le rôle des étouffoirs, des marteaux, fait faire des glissandi sur le clavier, dans les cordes avec et sans pédales (photo 2). Elle montre les différents clusters (jouer des grappes de son sur le clavier à l'aide de la paume de la main ou de l'avant-bras). Pour le moment la place de l'enseignante dans la topogenèse est importante quand elle dit « je vais vous montrer comment on fait les sons bizarres qu'on a entendus » (transcript 2, 19'30, ci-dessus et photo 2, ci-dessous).

\begin{tabular}{|l|l|}
\hline Photogramme 2 : exploration sonore à l'intérieur du piano, $\mathbf{1}^{\text {re }}$ séance du $\mathbf{2}^{\mathbf{e}}$ groupe \\
\hline $\begin{array}{l}\mathrm{P}: \text { Donc on récapitule, là on peut glisser comme ça (gras du } \\
\text { doigt }) \text {; glisser comme ça (sur les ongles) et toi Juliette quand tu } \\
\text { fais de la guitare tu fais comment? } \\
\text { E1: Je pince les ongles } \\
\mathrm{P}: \mathrm{Tu} \text { pinces les ongles... }\end{array}$ \\
\hline $\begin{array}{l}\text { P synthétise les nouveaux savoirs que les élèves ont entrevus en explorant le piano. Ensuite elle les oriente } \\
\text { par questionnement, en faisant référence à quelque chose de connu (la guitare) }\end{array}$
\end{tabular}

Cependant elle laisse libre court aux essais des élèves (synopsis 1) quand il s'agit pour eux, de s'approprier les outils avec les contraintes que cela implique (jouer dans les cordes, avec ou sans objet). Ils testent leur jeu pour retrouver ce qu'ils ont entendu. Ils connaissent déjà la notion d'intensité sonore (piano, forte), et celle de hauteur de son (grave, aigu). Ce sont les seules références qu'ils trouvent de leurs expériences passées dans l'ancien contrat de piano. 
Les élèves ont exploré plusieurs façons de jouer en vingt-deux minutes seulement (cf. minutage dans la colonne de gauche, synopsis 1, ci-dessous). P n'intervient que pour donner des ustensiles, elle laisse progressivement la place aux élèves (E représentant l'ensemble des élèves car nous n'avons pas réussi à distinguer qui faisait quoi).

\begin{tabular}{|l|l|}
\hline \multicolumn{2}{|l|}{ Synopsis 1 synopsis du travail sur les cordes } \\
\hline $30: 00$ & E : essai sur le piano de glissandi, sans pédale puis découverte des étouffoirs \\
\hline $32: 15$ & P : rapprochement avec l'écoute \\
\hline $33: 00$ & E gras du doigt \\
\hline $33: 40$ & E pincer \\
\hline $42: 00$ & E : frappe sur le bois \\
\hline $45: 50$ à & E : résonance dans le piano couvercle ouvert et pédale avec la voix \\
$47: 00$ & \\
\hline $50: 00$ à & P : donne des balles de mousse et de ping pong \\
$52: 00$ & \\
\hline
\end{tabular}

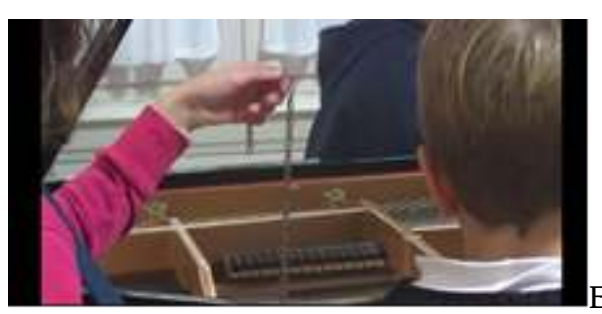
découvrent les accessoires mis à leur disposition: chaîne, balles, feuilles de papier, coquillages (photo 3).

Voici une deuxième rupture de contrat. Le contrat de piano précédent, celui qui les a guidés pendant un an (" joue les notes écrites sur la partition, avec les doigts et sans erreurs »), est rompu. On a le «droit» de faire des «fausses » notes et de toucher à tout le piano (sauf les étouffoirs), de faire des sons «bizarres ». Vu la force du précédent contrat, qui était devenu incorporé, il est nécessaire que ce nouveau contrat soit explicite : il faut chercher toutes les façons possibles de faire des sons.

Dans la phase 1, le milieu servait de référence auditive (avec l'enregistrement, l'ouïe et la parole). Il a évolué puisque dans la phase 2 l'enregistrement disparaît ${ }^{19}$ et l'instrument est proposé. Les élèves sont devenus analystes verbaux et sensitifs (transcript 1 et transcript du photogramme 2); puis explorateurs (sur l'instrument, photos 2 et 3 ; synopsis 1 ). Au milieu précédent s'ajoutent d'autres sens comme la vue, le toucher, le reste du corps, l'instrument de musique et les ustensiles.

59 P propose ainsi aux élèves une enquête sur l'instrument (photos 2 et 3 ; synopsis 1 ) qui doit leur permettre de commencer à se saisir de nouveaux possibles pour construire un nouveau « voir comme » dans le sens de Wittgenstein (Glock, 2003) ${ }^{20}$.

60 Cette réflexion nous incite à faire migrer le concept de dévolution exposé par Brousseau (2003, p. 5) de la façon suivante: "La dévolution consiste pour l'enseignant, non seulement, à proposer à l'élève une situation qui doit susciter chez lui une activité non convenue » (ici ce serait de jouer sur le piano autrement que sur le clavier), « mais aussi à faire en sorte qu'il se sente responsable de l'obtention du résultat proposé » (op. cit.). Le verbe sentir montre que l'élève peut ne pas être entièrement responsable du résultat mais que c'est le sentiment de responsabilité qui importe. Ce qui voudrait dire, qu'après plusieurs essais de sons, l'élève puisse les reproduire selon son choix en ayant le sentiment qu'il en a la responsabilité :

[...] Et qu'il accepte l'idée que la solution ne dépend que de l'exercice des connaissances qu'il possède déjà. L'élève accepte une responsabilité dans des 
conditions qu'un adulte refuserait puisque s'il y a problème puis création de connaissance, c'est parce qu'il y a d'abord doute et ignorance. C'est pourquoi la dévolution crée une responsabilité mais pas une culpabilité en cas d'échec. (Brousseau, 2003, p. 5)

61 La situation de ces cours est en ce sens " particulière » puisque le problème est de jouer avec d'autres gestes et de faire en sorte que ce qui paraissait bruit soit perçu comme son par l'agencement des différentes composantes sonores ${ }^{21}$. Ce que la professeure exprime ainsi : « entre bruit et son c'est que le bruit n'a pas de sens » (propos recueillis dans le email de janvier 2016).

Ici les conditions paraissent réunies : les élèves acceptent de se plier au jeu, essayant de trouver des sonorités différentes en prenant appui sur ce que $\mathrm{P}$ a fait entendre et a montré. La professeure se désengage progressivement. Elle occupe une position plus basse dans la topogénèse tout en ponctuant les séances par des propos qui commencent à institutionnaliser les savoirs comme : « bravo » (transcript 1, 18').

\section{2.b Construction d'une production musicale. Réinvestissement des nouvelles connaissances.}

63 Les séances suivantes comprennent toujours la phase de remémoration et celle du prolongement de l'invention musicale. Cependant, à la deuxième séance, les élèves ne se souviennent plus ou très peu de ce qu'ils ont inventé. $\mathrm{P}$ doit donc se référer aux feuilles sur lesquelles elle a pris soin de noter la production des élèves. Dans le transcript de la figure 8, la professeure demande à l'élève de se remémorer ce qu'elle avait fait la semaine précédente (en italique l'action).

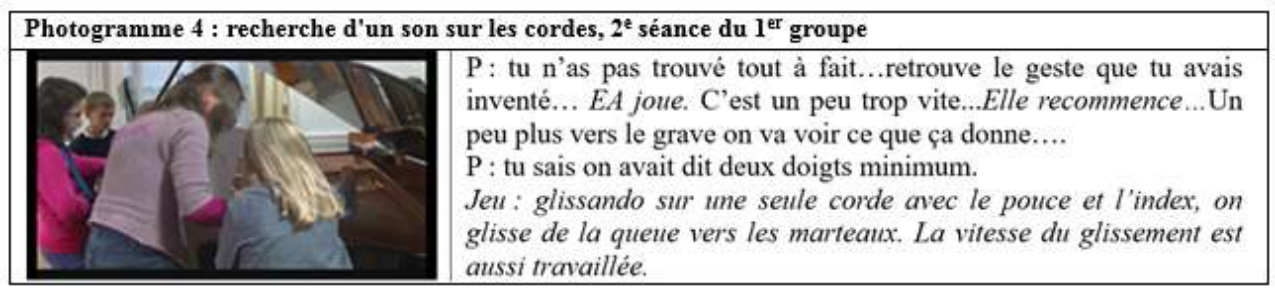

L'objectif de mémorisation des phases deux et trois de la première séance n'ayant pas été explicité, entraîne un ralentissement de la production musicale montrant que la mémorisation de nos actes, si elle n'est pas organisée ou instituée, n'intervient que partiellement (Lieury, 1995). Ce que l'élève nommée EA n'a pas saisi, c'est que la répétition du geste est décisive dans un tel apprentissage (Billeter, 2012 ; Menuhin, 1976). Dans les cours de piano dits «classiques », la répétition du geste est prégnante et fait partie du contrat. Il semble ici que la partie du présent contrat qui consiste à dire « on fait autre chose que... » a été aussi perçue comme "on n'a pas besoin d'apprendre ni de répéter ». C'est-à-dire que si la répétition n'est plus perçue comme une nécessité, les éléments contractuels disparaissent. Ce qui faisait partie d'un contrat antérieur explicite (retenir ce qui est fait en cours) est devenu implicite pour P mais pas pour EA. La contrainte gestuelle pour une réalisation du jeu pianistique, comme par exemple celle de jouer ici avec deux doigts, n'a pas été perçue, quant à elle, comme nécessaire à la réalisation du son recherché.

Cette recherche musicale conjointe entre P et EA a pour objectif de retrouver l'effet sonore voulu : « Un peu plus vers le grave, on va voir ce que ça donne », dit P. Au cours de cette remémoration, il existe une forme de dialogue qui s'établit de la façon suivante: $\mathrm{P}$ 
parle et EA joue, $\mathrm{P}$ commente avec des mots ce qu'elle vient d'entendre et EA modifie son jeu et le son ${ }^{22}$. C'est un exemple très dense de l'action conjointe élève/professeur où la topogénèse évolue : la professeure est très présente à nouveau, par nécessité.

L'expérience passée n'est pas ancrée. Son intégration nécessite de la renouveler ${ }^{23}$ et de la partager par le langage (transcription du photogramme 2). Dans le contrat initial qui était " cherchez des sons", s'insère momentanément un sous-contrat "retrouvons ensemble ce que tu avais trouvé seule » qui, cette fois, est explicité. Cependant, la dévolution qui était initiée lors de la première séance est en retrait par le fait que la professeure est obligée de reprendre un rôle actif : on voit ici le rapport entre topogénèse et dévolution. Cet aller et retour "absence-présence», illustre les différents topos. Cette structure topogénétique est physiquement visible: quand la professeure laisse les élèves en responsabilité de leur production musicale elle se recule (elle n'est plus dans le champ de la caméra, c'est pourquoi nous ne la verrons pas dans les photogrammes suivants) et quand elle intervient, elle revient près du piano (ci-dessus photo 4).

Cette première analyse a tenté de rendre visible l'action conjointe professeur/élève en partant des propositions de la professeure. Celle-ci ayant pour objectifs d'enseignement que les élèves soient capables de reconnaître et de reproduire une manière de jouer particulière propre à un style de musique, à partir d'une démarche d'observation et de reconnaissance de ses caractéristiques. Puis qu'ils se les approprient afin de les restituer pour inventer collectivement une musique.

Nous commençons seulement à entrevoir comment corporellement cela se fait. Nous proposons maintenant de voir comment l'élève incarne avec et dans son corps ce nouveau jeu pianistique. Orientons-nous vers une micro-analyse qui mette en rapport le bloc «son-style / jeu-esthétique » avec le geste correspondant. Nous nous focaliserons davantage sur les limites de ce que l'enseignant peut apporter, puisque dans l'enseignement d'un instrument de musique, on ne peut pas faire "à la place de », le corps de l'autre étant étranger à soi. Ce qui remet en question la notion de dévolution.

\subsection{Formes de travail rencontrées et retour au contrat initial : un geste correspond à un son prévisible}

Lors des expériences sonores de la phase 1 , les élèves ont testé le rapport qui existe entre un geste et un son qui lui correspond. Lors des phases suivantes, il y a une phase de mémorisation du geste et de sa représentation sonore, une prise de conscience de l'action du corps sur l'objet et de ses conséquences sonores.

\section{3.a Corps et objets pour une production collective}

\section{Travail sur la percussion}

71 Première forme de travail ci-dessous, photogrammes $5 a, b, c$ : un élève (en bleu foncé à gauche) fait des glissandi pendant que deux autres jouent avec les rebonds d'une balle de ping-pong, la pédale de résonance est enfoncée et maintenue. À ce moment du jeu, l'élève en blanc à droite se prépare à jouer une note et l'élève que l'on aperçoit (c) derrière celle qui est en rose, écoute en attendant son tour (c'est celle du photogramme 4). 


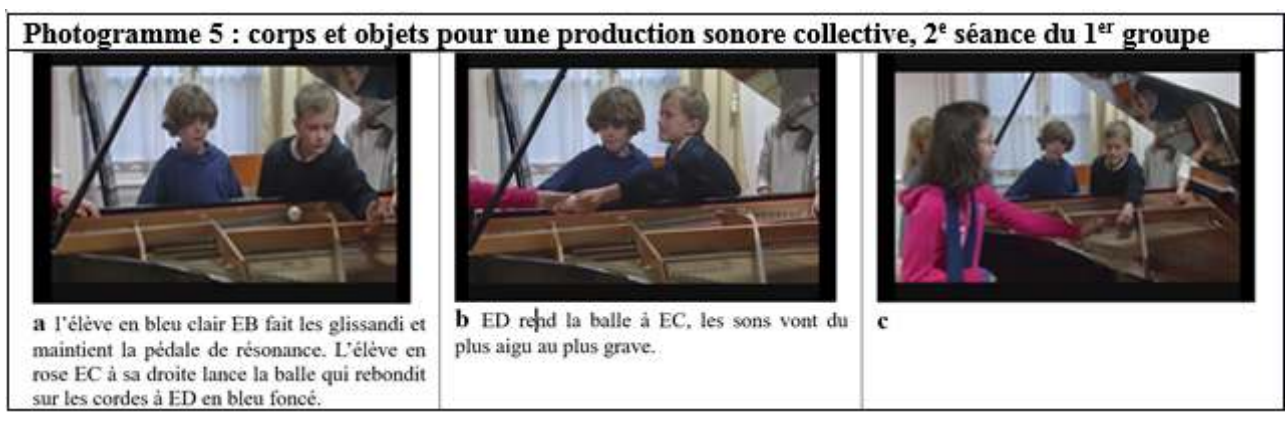

72 Dans cet exemple on distingue trois dimensions :

- les matériaux et leurs propriétés sonores et dynamiques ;

- l'action engendrée par la percussion d'un objet ;

- l'action conjointe des deux élèves.

La première dimension est le choix qu'ont fait les élèves à l'issue de la phase d'exploration (phase 1): la balle de ping-pong. Elle a été choisie pour le son qu'elle produit et par conséquent pour ses propriétés : matériau léger et fin, aux propriétés rebondissantes produisent un son dont les paramètres sont le timbre, l'intensité et l'effet percussif. Nous pouvons qualifier le son comme "clair", aléatoire et très « rebondissant ».

L'utilisation de cet objet permet aux élèves de percevoir d'une part, le rôle de l'objet qui percute la corde, parce qu'il le voit et d'autre part, le rôle de la matière qui percute. Ils peuvent ainsi comprendre qu'en fonction de la matière, du poids de l'objet et de la vitesse de percussion, un son sur une corde peut être différent. Ils se rendent compte aussi que la vitesse de percussion dépend de l'action du bras, dans son amplitude et dans sa vitesse. C'est la deuxième dimension.

La troisième dimension est la production d'un seul son à deux. Avec la balle de ping-pong, les élèves construisent ensemble le son, mais aussi des cellules rythmiques générées par le lancement de la balle et par la façon dont elle est rattrapée. Associé à l'action de l'autre élève qui fait des glissandi sur le piano, et malgré le rebondissement aléatoire de la balle, cela engendre une polyphonie et une polyrythmie choisies et coordonnées. La professeure n'intervient pas. La clause proprio motu est complètement réalisée, les élèves sont responsables l'un de l'autre et de leur production sonore collective. Le processus de dévolution est bien avancé.

\section{3.b Utilisation des bras et implication de tout le corps}

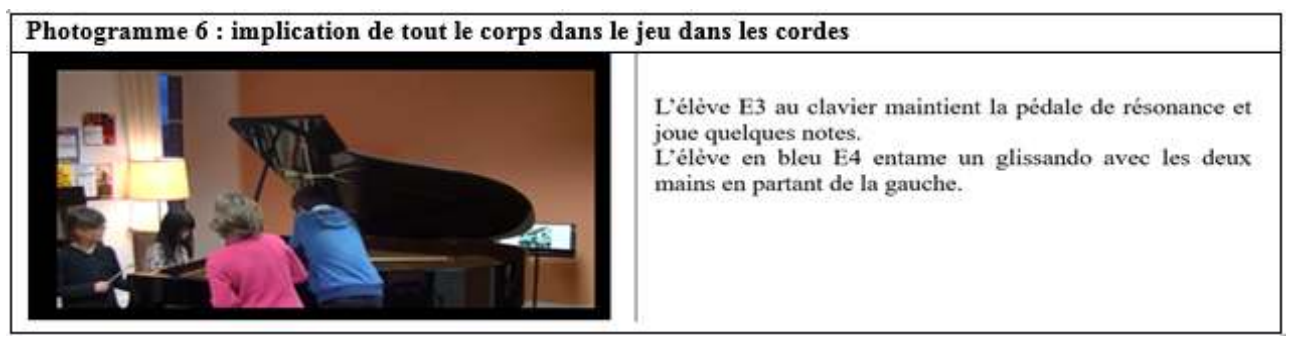

Le photogramme 6, ci-dessus, est un instantané extrait de l'audition pendant laquelle les élèves du deuxième groupe observé jouent en public leur invention musicale à quatre. Chacun a un rôle qu'ils se sont collectivement attribué au cours des cinq séances précédentes. Le public est composé d'autres élèves et de trois professeurs. Deux des 
élèves que nous voyons sur la photo viennent de jouer et se retirent momentanément parce que, à ce moment du morceau joué, leur rôle s'amoindrit ; il ne reste que l'élève en bleu (E4) et l'élève au clavier (E3). E3 étant l'élève dont nous avions rapporté les propos au moment de la phase d'exploration dans la transcription 1. Revenons au photogramme actuel (6). E4 est monté sur un petit escabeau. Il « plonge » dans le piano à partir de la voûte plantaire jusqu'à la main, en passant par les jambes, le bassin, le torse, l'abdomen, le dos, les épaules.

Cette expérience permet aux élèves de se rendre compte que tout le corps est impliqué et l'ancrage au sol est important pour le jeu pianistique. Il s'agit de pouvoir, le cas échéant, transférer son poids d'une partie du corps sur l'autre (même si cela n'est pas toujours visible) pour pouvoir modifier l'intensité sonore du jeu sur le clavier ou ailleurs.

Deuxième forme de travail : nous avons vu (photo 6) que, pour faire un glissando sur toute l'étendue du clavier, pour envoyer et rendre la balle, les élèves doivent utiliser le bras. Ce mouvement part des épaules.

L'exemple suivant (photo 7) est dans le prolongement du précédent (photo 6). Le son est encore plus directement lié au geste du bras et du corps. Les quatre vignettes (a, b, c, d) s'enchainent sur dix secondes.

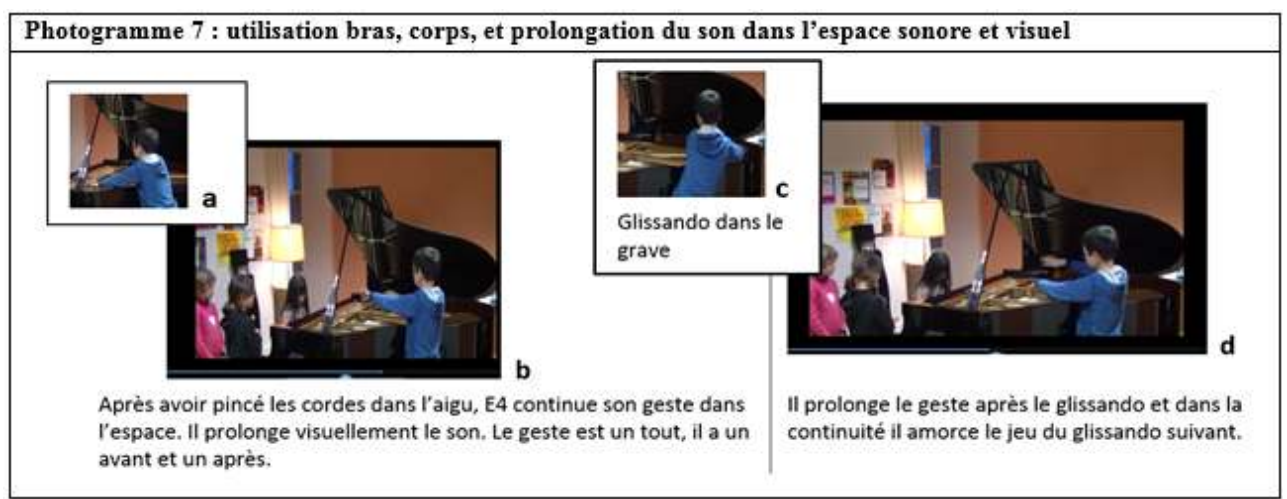

Quand E4 pince les cordes (a), ci-dessus, il amorce un geste et le prolonge (b) après le moment où il initie le son. Ce geste se poursuit jusque dans le grave du piano où il fait un glissando (c) dans le prolongement du son et du geste précédents ( $a$ et b). Lorsqu'il a fini le glissando dans le grave (c), il retourne vers les cordes aigües en prolongeant son geste (d). Pendant ce temps, E3 maintient la pédale de résonance enfoncée et égraine quelques notes sur le clavier. E4 est dans une expérience esthétique liée à un geste qui, pour que la résonance de la corde ne soit pas interrompue, ne doit pas être arrêté. Si le musicien a l'intention de jouer un autre son en relation avec le précédent, la fin du premier geste du premier son (a) deviendra le début du deuxième geste (b) et donc l'amorce du deuxième son (c), comme la main d'une harpiste après le pincement de corde. Ici le son de E4 dépend du maintien de la pédale de $\mathrm{E} 3$.

81 Dans cette chaine expérientielle travaillée pendant six séances et dont l'aboutissement est l'audition partiellement décrite ci-dessus, l'élève évolue dans un milieu-soi (BatézatBatellier, 2012 ; Forest \& Batézat-Batellier, 2013). Comme un savoir opérationnel, il va faire un geste qu'il va mémoriser : il va faire l'expérience puis prendre connaissance ${ }^{24}$ que le geste de son propre corps est associé à un son, pour ensuite être capable de le reproduire quand il en aura besoin. Peut-être avons-nous là une illustration de ce que Dewey (1938/1993) nomme assertibilité garantie qui désigne ce que d'autres nomment la vérité, la connaissance ou la croyance, montrant que sa valeur n'a de sens qu'en 
découlant d'une enquête particulière. Les idées sont un prolongement de nos actions, de nos expériences. L'enquête se transforme et se confirme dans l'expérience et amène une solution à un problème donné.

Reprenons ci-dessous la transcription du photogramme 4 (recherche d'un son) où $\mathrm{P}$ insiste sur la vitesse du geste et sur le poids du bras sur la corde, en voici le transcript.

\begin{tabular}{|l|l|l|}
\hline \multicolumn{2}{|l|}{ Transcript 3 transcript du photogramme 4 } \\
\hline $0: 06: 05$ & $\mathrm{P}$ & $\begin{array}{l}\text { tu n'as pas trouvé tout à fait...retrouve le geste que tu avais inventé... Un peu plus vers le grave } \\
\text { on va voir ce que ça donne...C'est pas mal, encore une fois....quand tu vas trop vite, tu sais on } \\
\text { avait dit deux doigts minimum... }\end{array}$ \\
\hline & $E A$ & joue. \\
\hline $\mathrm{P}$ & Un peu plus fort \\
\hline & $E A$ & joue Si t'es légère ça fait ça... \\
\hline & $P$ & joute, si t'appuies un peu plus \\
\hline & $P$ & joue, \\
\hline & $E A$ & joue, \\
\hline$P$ & Voilà. Essaie sur la corde grave là \\
\hline & $E A$ & joue \\
\hline & $\mathrm{P}$ & Non bon Donc un peu plus fort, un peu plus. En fait il faut que tu le vois là l'éclair. \\
\hline
\end{tabular}

$\mathrm{P}$ a recours à une représentation visuelle. Elle associe l'image de l'éclair utilisée dans l'histoire à la vitesse de son geste et à la notion de poids qui doivent permettre l'amplification du son. Elle montre comment ça doit sonner, presqu'en même temps qu'elle parle. Les élèves associent l'image, le son, le geste et la parole.

En lui demandant de retrouver le son et le geste de la séance passée, $\mathrm{P}$ demande à EA de recréer, de revivre, de retrouver les sensations corporelles et auditives qu'elle a « intériorisées » lors « d'expériences » précédentes. En les recherchant, elle est obligée de faire appel à sa mémoire. C'est ce qui est appelé communément « l'écoute intérieure ». On suppose ainsi que quand un son est produit, il est susceptible d'être mémorisé. Quand il est mémorisé chez un individu, ce dernier peut l'imaginer, et en réactualiser l'expérience dans toutes ses dimensions corporelles. Ces expériences sont associées à d'autres sensations, visuelles, affectives et/ou kinesthésiques. En les retrouvant et en les extériorisant, lors d'une exécution, les élèves sont dans un processus de recréation de l'expérience vécue (Dewey, 1934/2005), car un geste humain ne peut pas être reproduit à l'identique (Billeter, 2012).

C'est ce qui se passe ici. Quand $\mathrm{P}$ parle de l'éclair, cela réfère à un son connu, les élèves cherchent donc à produire des sons qui «illustrent » l'éclair. À un moment donné, ils décident que tel son est le son approprié, ils l'ont exploré avec leur corps, avec des gestes. Ils ont fait implicitement des liens de cause à effets. Cette association son/mot permet aussi de retrouver le geste.

\section{3.c Sensations digitales et visualisation de la vibration de la corde}

Dans le photogramme 8, l'élève ayant expérimenté une façon de jouer en bloquant la corde, le reproduit ci-dessous.

\begin{tabular}{|l|l|}
\hline Photogramme 8 : travail sur le toc, clavier et cordes, $2^{2}$ séance du $\mathbf{1}^{\text {er }}$ groupe \\
\hline $\begin{array}{l}\text { EE joue une note et bloque la corde avec un doigt. } \\
\text { Le son ainsi obtenu est court et étouffé, et } \\
\text { ressemble plus à un son de percussion. }\end{array}$
\end{tabular}


Ci-dessus, EE sent la vibration de la corde avec son doigt et sent que si elle appuie davantage, le son s'éteint. Elle comprend alors mieux comment agissent les étouffoirs et l'utilisation de la pédale de résonnance ( $1 / 2$ pédales etc.). Il est aussi possible de provoquer des harmoniques en effleurant la corde une fois qu'elle a été jouée avec la pédale maintenue. Dans la séance décrite ici il n'en a pas été question, mais dans d'autres séances observés cette technique est utilisée.

Dans le photogramme 9, en posant une feuille ou un coquillage sur les cordes, la vibration de la corde est plus visible. On remarque alors que la percussion sur la corde génère une vibration qui produit un son.

\begin{tabular}{|l|l|}
\hline Photogramme 9 : vibration d'une feuille sur les cordes, $\mathbf{2}^{\text {eme }}$ séance du $\mathbf{1}^{\mathrm{er}}$ groupe \\
\hline & $\begin{array}{l}\text { La pédale de résonance est maintenue } \\
\text { enfoncée, EE tape sur les cordes avec la } \\
\text { paume de la main gauche, une feuille et un } \\
\text { coquillage sont posés sur les cordes jouées, ils } \\
\text { vibrent avec les cordes. On entend un } \\
\text { bruissement de feuille, on la voit bouger. }\end{array}$ \\
\hline
\end{tabular}

9 Cette expérience est importante. Pour les pianistes, en dehors de l'effet sonore voulu, elle permet de comprendre qu'un son a un début et une fin, qu'il peut être généré de façon différente, qu'il est le résultat de vibrations qui ne sentent pas (ou très peu) lorsque l'on est au clavier.

Dans cette partie, nous avons souhaité montrer comment le corps est impliqué dans l'apprentissage d'un instrument de musique. Il est impliqué comme étant à l'origine d'une expérience sonore, qui par essence est aléatoire. Par une attention particulière au son et aux sensations corporelles puis par la répétition du geste qui lui correspond, les élèves tentent de reproduire et d'atteindre l'effet voulu, dans le but de construire une production musicale, dans un style musical donné. $\mathrm{P}$ est intervenu au début (photogramme 2, transcript 1) et l'on a vu, ici, qu'elle se retire progressivement, (transcript 2, synopsis 1 puis photo 4) laissant à chaque élève le soin de prendre en charge sa part de responsabilité individuelle dans cet acte collectif (photo 5 puis photos 6 et 7).

\section{Conclusion}

Quand la recherche d'une esthétique du son devient source d'expérience et d'apprentissage, elle s'appuie sur des sensations corporelles nouvelles. Dans le cas de ce travail qui aborde une esthétique particulière, issue en particulier de la musique dite " contemporaine ", le fait de rechercher un univers sonore met l'élève en situation d'expérience. Cela l'oblige à rompre avec la familiarité qu'il a déjà d'un jeu pianistique de clavier dit "classique». La proposition d'un nouveau milieu rompt avec le contrat didactique habituel pour en inviter un nouveau. Elle met les élèves en situation de déséquilibre (comme lorsqu'ils écoutent les enregistrements). On assiste alors dans la dialectique contrat-milieu à la recherche d'un nouvel équilibre qui oblige chaque élève à avoir une nouvelle responsabilité dans la production sonore collective. Celle-ci organisée, devient un discours, avec des phrases et des respirations. 
92 Le fait de chercher à produire des sons et non des notes, d'avoir accès à différentes parties du piano, ouvre les possibles. Par l'intermédiaire du professeur, les élèves choisissent le matériau sonore le plus adéquat. Ils sont incités à utiliser leur corps, pour viser une certaine forme d'efficacité dans le rapport au geste et au son. Le piano " démécanisé » leur donne alors l'occasion de chercher des timbres, de créer des expériences sonores par une expérience gestuelle personnelle.

Dans le collectif, l'action du professeur (en tant qu'instance) est subtile, laissant à l'individu une responsabilité propre. L'exemple corporel ne pouvant être que personnelle, ce sera le son en tant que rétroaction du milieu qui donnera des indications au professeur sur le degré d'intégration du geste musical de l'élève. Ce seront ces indications qui orienteront l'action conjointe professeur/élèves et élève/élève, dans le processus de dévolution. Ce concept en musique pourrait être discuté dans le sens où, un professeur ne peut pas « faire » à la place de l'élève (en tant qu'instance), car il n'a pas accès au corps de l'élève. Il ne peut que montrer une partie du geste, celle qui est extérieure à lui. Par contre le son sera perçu totalement. L'élève ne peut alors que "faire-comme" son professeur et là encore ce serait le son qui, par rétroaction, lui donnerait des indications pouvant l'aider à évoluer dans ce milieu. Sachant aussi que chaque corps est différent et par là propose une partie du milieu particulière à chaque élève. C'est la musique qui engage l'élève dès le début de son apprentissage dans un processus de dévolution, et pas seulement la professeure.

Avoir vécu une expérience esthétique où il faut être plusieurs pour produire un son, pour œuvrer à une production musicale et à une interprétation co-construite, peut s'apparenter aux problèmes que se posent les compositeurs d'une part, et les interprètes d'autre part, quand ils cherchent à partager quelque chose avec un public. Quand il écrit une œuvre, le compositeur dévolue « virtuellement » à l'interprète la responsabilité de la présenter à un public et de la rendre vivante. Dans cette nouvelle approche du piano, les élèves de notre étude ont acquis certaines spécificités décrites dans le paragraphe sur l'interprétation. C'est-à-dire qu'ils ont acquis des connaissances d'un style particulier de musique dans une esthétique déterminée ; une technique leur permettant, à leur niveau, de jouer «à la manière de ", et une capacité à rendre intelligible leur discours musical et à le produire en public. Nous pouvons dire qu'ils sont dans une démarche d'interprète de leur propre production musicale. Ils ont une responsabilité vis-à-vis d'eux-mêmes, de leur apprentissage. Du point de vue topogénétique, la professeure et le son musical ont un rôle apparenté et intiment liés. Ainsi au moment de leur jeu en public, lorsqu'ils sont seuls avec leur musique, les élèves sont dans une étape importante d'un processus de dévolution que semble permettre l'enseignement que nous avons décrit.

\section{BIBLIOGRAPHIE}

Arnold, D. (1983/1993). Dictionnaire encyclopédique de la musique (M.-S. Pâris, Trad.) Paris : Robert Laffont. Badura-Skoda, P., \& Badura-Skoda, E. (1974). L'art de jouer Mozart au piano. Paris : Buchet/Chastel. 
Batézat-Batellier, P. (2012) Un apprentissage particulier dans une école élémentaire : l'instrument de musique en orchestre comme exemple d'une approche en didactique de la musique. (Mémoire de Master 2, Rennes 2). Consulté sur http://cread.espe-bretagne.fr/spip.php?article638.

Bazin, J. (2000). L'anthropologie en question : altérité ou différence ? Dans Y. Michaud (Éd.), Qu'est-ce que la société ? (pp. 78-88). Paris : Odile Jacob.

Beaugé, P. (2002). Un savoir musical : la notion de hauteur. Journal de Recherche en Education Musicale, pp. 55-84.

Bernard, M. (1995). Le corps. Paris : Seuil.

Bourg, A. (2008). Didactique de la musique : apports d'une approche comparatiste-Etude des notions de transposition didactique et d'organisation praxéologique pour l'enseignement/ apprentissage du piano. Éducation et Didactique, II(1), pp. 69-88.

Bourg, A., \& Guillot, G. (2015, mars). La didactique de la musique au sein des didactiques disciplinaires : emprunts et comparatismes. Recherche En Éducation Musicale, 32. Récupéré sur http://www.mus.ulaval.ca/reem/sommaire_courant.htm

Brousseau, G. (1998). Théorie des situations didactiques. Grenoble : La Pensée Sauvage.

Brousseau, G. (2003, Février 25). Glossaire de quelques concepts de la théorie des situations. Consulté le 2014, sur http://daest.pagesperso-orange.fr/guy-brousseau/textes/Glossaire_Brousseau.pdf Canonne, C. (2015). Le piano préparé comme « esprit élargi » de l'improvisateur : Une étude de cas autour du travail récent d'Ève Risser. Consulté sur http://www.musimediane.com/numero8/ CANONNE/

Caullier, J. (2012). La condition d'interprète. Consulté le 2015, sur DEMéterv : http:// demeter.revue.univ-lille3.fr/lodel9/index.php?id=205

Chevallard, Y. (1992). Concepts fondamentaux de la didactique : perspectives apportées par une approche anthropologique. Recherches en didactique des mathématiques, 1 (12), pp. 73-112.

Chevallard, Y. (1985/1991). La transposition didactique. Du savoir savant au savoir enseigné. Grenoble : La Pensée Sauvage.

Chevallard, Y. (Sous Presse). La TAD et son devenir : rappels, reprises, avancées. Dans G. Cirade, \& al, Evolutions contemporaines du rapport aux mathématiques et aux autres savoirs à l'école et dans la société. Actes du 4ème congrès international sur la TAD.

Cowell, H. (1930/1996). New musical resources. Cambridge : Cambridge University Press.

Cowell, H. (2015). The fairy answer [Enregistré par F. Bongelli]. Consulté sur https:// www.youtube.com/watch ?v =D3CPrdI2FjM

Crumb, G. (2004). Makrokosmos [Enregistré par M. Leng Tian]. Sur Makrokosmos I \& II : 24 fantasy pieces after the Zodiac for amplifies piano. New Yok : Mode Records.

Dewey, J. (1934/2005). L'art comme expérience. (J.-P. Cometti, Trad.) Paris : Gallimard. Coll.Folio Essais.

Dewey, J. (1938/1993). Logique : la théorie de l'enquête. (G. Deledalle, Trad.) Paris : PUF.

Donington, R. (1998). Interpretation. Dans S. Sadie, The new Grove dictionnary of Music and Musicians (Vol. IX, p. 276). Londres : Mac Millan Publishers Limited.

Fleck, L. (1934 / 2005). Genèse et développement d'un fait scientique. Paris : Les Belles Lettres. 
Forest, D. (2009). Agencements didactiques, pour une analyse fonctionnelle du comportement non-verbal du professeur. Revue Française de Pédagogie, 165, pp. 77-89. Récupéré sur http:// rfp.revues.org/1108

Forest, D., \& Batézat Batellier, P. (2013). Apprentissage d'une pratique instrumentale en orchestre à l'école : une approche didactique. Education \& Didactique, VII (3), pp. 79-96.

Foucault, M. (1963). Naissance de la clinique. Une archéologie du regard médical. Paris : PUF.

Ginzburg, C. (1925/1989). Traces. Racines d'un paradigme indiciaire. Dans Mythes, emblèmes et traces. Morphologie et histoire (pp. 149-180). Paris : Flammarion.

Glock, H. J. (2003). Dictionnaire Wittgenstein. Paris : Gallimard.

Goodman, N. (1984/1996). L'art en théorie et en action. Paris : Gallimard

Greilsammer, D. (2015, mars 3). Prépared piano. Consulté sur : https://www.youtube.com/watch ? $\mathrm{v}=\mathrm{kc} 3-\mathrm{C} 7 \mathrm{Lnzh} 0$

Griffiths, P. (1992). Brève histoire de la musique moderne. De Debussy à Boulez. France : Fayard.

IRCAM. (2010). Analyse musicale d'interprétations enregistrées. Paris. Consulté sur http:// www.apm.ircam.fr/performance_analysis

Kurtág, G. (1973-2010). Játékok. Budapest : EMB.

Leutenegger, F. (2000). Construction d'une "clinique" pour le didactique. Une étude des phénomènes temporels de l'enseignement. Recherche en Didactique des Mathématiques, 20 (2), pp. 209-250.

Levi-Strauss, C. (1949). Structures élémentaires de la parentée. Paris : PUF.

Lieury, A. (1995). Mémoire des images et double codage. L'année Psychologique, 95 (4), pp. 661-673.

Menuhin, Y. (1976). Voyage inachevé. Autobiographie. Paris : Seuil.

Mialaret, J.-P. (1997). Explorations musicales instrumentales chez le jeune enfant. Paris : PUF.

Mili, I. (2012). Créativité et didactique dans l'enseignement musical. Education et Francophonie, XL, pp. 139-153.

Nattiez, J.-J. (1987). Musicologie générale et sémiologie. Paris : Christian Bourgois.

Poirier, A. (2015). George Crumb. Consulté sur Ircam : brahms.ircam.fr/george-crumb

Pressing, J. (1984). Cognitive process in improvisation. Dans W. Crozier, \& A. Chapman, Cognitive Processes in the Perception of Art (pp. 345-363). Amsterdam : Elsevier.

Ramaut-Chevassus, B. (1998). Musique et postmodernité. Paris : PUF.

Rettel, G. (2015, février). Etude de la place de la musique dans les bibliothèques de la ville de Paris et l'avenir de la médiatèque musicale de Paris. Récupéré sur http://www.enssib.fr/bibliothequenumerique/documents/65333-etude-sur-la-place-de-la-musique-dans-les-bibliotheques-de-laville-de-paris-et-l-avenir-de-la-mediatheque-musicale-de-paris.pdf

Sadie, S. (1998) The new Grove dictionnary of Music and Musicians. Londres : Mac Millan Publishers Limited.

Sensevy, G. (2011). Le sens du savoir. Bruxelles : De Boeck

Sensevy, G. (2011a). Comprendre l'action didactique. Méthode et jeux d'échelle. Dans G. sensevy, Le sens du savoir. Bruxelles : De Boeck. Récupéré sur

http//python.espe-bretagne.fr/sensevy/sensdusavoir/ 
Sensevy, G. (2011b). Le sens du savoir. Consulté sur http://python.bretagne.iufm.fr/sensevy/ sensdusavoir/LeSensDuSavoirChap6.pdf

Sensevy, G., \& Mercier, A. (2007). Agir ensemble. L'action didactique conjointe du professeur et des élèves. Rennes : Presses Universitaires de Rennes.

Tavan, C. (2003, février). Les pratiques culturelles : le rôle des habitudes prises dans l'enfance. Insee première. Récupéré sur www.insee.fr

Verret, M. (1975). Le temps des études. Paris : Honoré Champion.

Wilheim, A. (s.d.). György Kurtág, Jatekok. Paris. Consulté sur

http://brahms.ircam.fr/works/work/9775/

\section{NOTES}

1. Sachant que la population française écoute majoritairement de la musique dite "classique ", du jazz et du rock, musiques principalement tonales (Rettel, 2015). Sachant que les différences de pratiques culturelles varient en fonction de la CSP, du niveau d'étude des parents et du groupe d'âge (Tavan, 2003).

2. Mais qui est désignée comme moderniste par Griffiths (1992), bien que Ramaut-Chevassus (1998, p. 5-10) explique qu'il y a une ambiguïté dans les appellations entre «moderne », " postmoderne » et « contemporain ».

3. Professeure de piano, titulaire dans un conservatoire à rayonnement régional, ayant vingt-six d'expérience.

4. Entretiens avec la professeure, 16-01-2016.

5. Introduit le cluster dès 1912 (Arnold, 1983/1993, p. 559), Cowell (1930/1996, p. 111-139) dans l'édition commentée par D. Nicholls en explique la genèse. Les clusters sont une continuité des accords parfaits de l'harmonie classique par le fait qu'ils sont construits comme une succession de tierces et que, si l'on continue cette succession jusqu'à revenir à la fondamentale et que l'on regroupe toutes les notes ainsi obtenues, on obtient un agrégat de son.

6. Entretiens avec la professeure, 16-01-2016.

7. Entretiens avec la professeure, 16-01-2016.

8. «Le choix des œuvres et des compositeurs est justement lié aux sons que l'on va utiliser après » (entretiens avec la professeure, ibid.).

9. Cf. entretien avec la professeure du 16-01-2016.

10. Le glissando est une action des doigts qui glissent sur le clavier ou sur les cordes de grave vers l'aigu ou de l'aigu vers le grave.

11. Pour le triplet des genèses voir Sensevy, G., Mercier, A. et Schubauer-Leoni, M.L. (2000). La mésogénèse est la genèse du milieu didactique ; la topogénèse permet de montrer quel est le partage des tâches du professeur et des élèves dans l'avancée du savoir sur l'axe du temps et le partage de responsabilité de chacun dans les apprentissages ; la chronogénèse est la disposition du savoir sur l'axe du temps.

12. Cela rejoint d'idée de Szendy, (2001, p. 123) selon lequel «ce sont les écouteurs qui font la musique [...] ». Le créateur étant aussi un « écouteur ».

13. Dans le sens où l'improvisation libre n'a pas de référent et est conçue en temps réel (Canonne, 2015). Dans cette étude, l'improvisation demandée aux élèves fait référence à une écoute antérieure à l'acte musical.

14. Que l'on peut considérer comme un « déjà-là » du contrat didactique. 
15. Pour le piano la hauteur du son et la justesse étant prédéfinies on s'intéresse à la qualité du son.

16. D'après une des définitions du Centre National de Ressource Textuelles et Lexicale: exploration « Action d'appréhender quelque chose par les sens pour en connaître les qualités. »

17. «L'ethnographie consiste dans l'observation et l'analyse de groupes humains considérés dans leur particularité et visant à la restitution, aussi fidèle que possible de la vie de chacun d'eux.» (Lévi-Strauss, 1949).

18. Dans ce conservatoire, le contenu des cours de FM n'est pas écrit dans un projet pédagogique, cependant un tableau des contenus enseigné existe en notre possession, et cette affirmation est confirmée lors d'entretiens informels entre les professeurs de FM et nous.

19. Même si d'une certaine façon nous pouvons supposer qu'il reste dans la mémoire des enfants.

20. En le proposant « musicalement » on peut dire un nouvel « entendre comme».

21. Nous pourrions entrer dans une discussion son/bruit qui est, par ailleurs, communément débattue mais comme nous souhaitons rester au plus près de ce qui se dit en cours, nous resterons sur l'approche de Nattiez $(1987$, p. 73$)$ : « La distinction son/bruit n'a pas de fondement physique stable et [...] l'utilisation de ces deux concepts est, dès le départ, culturalisée ».

22. On assiste au « double codage » de Paivio décrit par Lieury (1995) qui associe des mémoires sensorielles aux mémoires lexicales et sémantiques

23. Cf. Mialaret (1997) qui montre comment la répétition des gestes a un rôle structurant - même si à chaque répétition il y a variation, mais aussi parce qu'il y a variation, comme le précise Dewey (1934) - et permet à l'enfant de construire sa propre expérience.

24. Ainsi que le ferait un bébé entre 6 mois et deux ans qui se rend compte que le pied qu'il voit et qu'il attrape pour s'amuser est bien le sien. Il se rend compte que le corps de sa mère et le sien sont deux corps distincts (Bernard, 1995).

\section{RÉSUMÉS}

L'article porte sur des cours collectifs où des élèves-pianistes en conservatoire de huit à dix ans expérimentent une approche de la musique contemporaine de type " piano préparé ». Il s'agit de montrer comment la recherche d'une esthétique musicale inconnue des élèves est le prétexte à la démarche d'interprète, et comment le professeur dévolue aux l'élèves la responsabilité de l'acte d'interprétation, termes que nous définirons. Nous verrons comment, l'expérience esthétique de la recherche d'un «son" s'ancre ou non corporellement. Les données recueillies sont principalement constituées de films vidéo. Elles sont abordées dans une perspective anthropologique dans le cadre d'une théorie de l'action conjointe en didactique avec méthodologie de type ethnographique. Est précisé l'usage des concepts de contrat, milieu, équilibration didactique et dévolution.

\section{INDEX}

Mots-clés : TACD, dévolution, apprentissage, contrat, collectif 
AUTEUR

PASCALE BATÉZAT-BATELLIER

CREAD, EA 3875 